\title{
Resonant Production of Scalar Diquarks at the Next Generation Electron-Positron Colliders
}

\author{
André Gusso * \\ Instituto de Física Teórica, Universidade Estadual Paulista, \\ Rua Pamplona 145, 01405-900 São Paulo - SP, Brazil
}

\begin{abstract}
We investigate the potential of TESLA and JLC/NLC electron-positron linear collider designs to observe diquarks produced resonantly in processes involving hard photons.
\end{abstract}

\section{INTRODUCTION}

Diquarks are amongst those particles whose features make them worth to be studied carefully. Like leptoquarks and dileptons, those particles are naturally predicted by many interesting extensions of the standard model as well as are invoked in other models in order to solve some of the most puzzling problems found in particle physics. These particles, also known as biquarks and duarks, carry baryon number $B= \pm 2 / 3$ and lepton number $L=0$, are colored and interact with quarks but not with leptons, and have integer spin.

Scalar diquarks are predicted in many Grand Unified Theories (with or without supersymmetry) [1] and models of composite particles [2], are invoked as an essential ingredient for a model that evades the strong $C P$ problem [3] and in [4] a class of models containing scalar diquarks is considered that can generate large $C P$ conserving and violating contributions to double Cabibbo suppressed decays without affecting $D^{0}-\overline{D^{0}}$ mixing. Vector diquarks are predicted by models of composite particles [2].

Diquark production was already analyzed for $e^{+} e^{-}, e p, p \bar{p}$ and $p p[5-8]$ colliders. They are expected to be easily observable at LHC [8] through their resonant production followed by the decay to two jets. Scalar and vector diquarks with masses up to approximately $10 \mathrm{TeV}$ could be observed, depending on their couplings to the quarks. If relatively light diquarks are observed they can also be expected to be on the reach of the next generation linear colliders, whose center of mass energy ranges from $500 \mathrm{GeV}$ up to $1 \mathrm{TeV}$ in the most conservative designs, like TESLA [9] and JLC/NLC [10]. The best channel for their observation on these machines would be, in principle, the pair production of diquarks. However, this channel is only useful if the diquark masses are smaller than $\sqrt{s} / 2$. This is a great limitation per se, and becomes more problematic when the present experimental limits on $E_{6}$ superstring inspired model scalar diquarks mass is taken into account, $290<M_{\phi_{E_{6}}}<420 \mathrm{GeV}[11]$.

\footnotetext{
*e-mail:gusso@fisica.ufpr.br
} 
In order to overcome this limitation we study the possibility of observing diquarks produced resonantly through the process depicted in Fig. 1. The resonant production of new particles through higher-order processes has already shown its usefulness as shown, for example, in [12] for the resonant production o dileptons. In this article we will focus on the production of scalar diquarks.

This article is organized as follows. In Sect. II we introduce the effective Lagrangians relevant to our analysis, and other theoretical and computational tools required to determine the cross-section for the process depicted in Fig. 1. In Sect. III we analyze the relevant background. In Sect. IV the results for the significance of the signal over background are presented. Finally, we conclude in Sect. V.

\section{SIGNAL}

Each extension of the standard model containing scalar diquarks we referred to in the Introduction has its won Lagragangian coupling diquarks with quarks. Yet, other models can be created and contain other Lagrangians. To take into account both the existing models and the ones that can be devised in the future we consider the most general $S U(3)_{c} \times S U(2)_{L} \times U(1)_{Y}$ invariant Lagrangian coupling scalar diquarks with the quarks of the standard model. The coupling of diquarks to other bosons and with itself are of no relevance here. Following Ref. [13], the possible scalar diquarks are $S U(3)$ triplets and sextets (In what follows, $i, j$ and $k$ are $S U(3)$ indices while $\alpha$ and $\beta$ are $S U(2)$, and family indices are omitted)

$$
\begin{aligned}
& S^{i}(3,1,-1 / 3), S^{\prime i}(3,1,-4 / 3), S^{\prime \prime i}(3,3,-1 / 3), S^{\prime \prime \prime i}(3,1,2 / 3) \text {, } \\
& S^{i j}(6,1,1 / 3), \quad S^{\prime i j}(6,3,1 / 3), \quad S^{\prime \prime i j}(6,1,4 / 3), S^{\prime \prime \prime} i j(6,1,-2 / 3) \text {, }
\end{aligned}
$$

where the $S U(3)_{c} \times S U(2)_{L} \times U(1)_{Y}$ multiplicities are given in parenthesis. Their couplings to the quark singlets $u$ and $d$, and doublets $q$ are given by

$$
\begin{aligned}
& \mathcal{L}_{S}=\lambda_{1} S^{i} \epsilon_{i j k} \epsilon_{\alpha \beta} \overline{q_{R}^{c}} \alpha j q_{L}^{\beta k}+\lambda_{2} S^{i} \epsilon_{i j k}{\overline{u_{L}^{c}}}^{j} d_{R}^{k}+\lambda_{3} S^{\prime i} \epsilon_{i j k}{\overline{u_{L}^{c}}}^{j} u_{R}^{k} \\
& +\lambda_{4} S^{\prime \prime i} \epsilon_{i j k}{\overline{q_{R}^{c}}}^{\alpha j}(\epsilon \tau)_{\alpha \beta} q_{L}^{\beta k}+\lambda_{5} S^{\prime \prime \prime} i \epsilon_{i j k}{\overline{d_{L}^{c}}}^{j} d_{R}^{k}+\lambda_{6} S^{i j} \epsilon^{\alpha \beta} \bar{q}_{L \alpha i} q_{R \beta j}^{c} \\
& +\lambda_{7} S^{i j} \bar{d}_{R i} u_{L j}^{c}+\lambda_{8} S^{\prime i j} \bar{q}_{L \alpha i}(\tau \epsilon)^{\alpha \beta} q_{R \beta j}^{c}+\lambda_{9} S^{\prime \prime i j} \bar{u}_{R i} u_{L j}^{c}+\lambda_{10} S^{\prime \prime \prime i j} \bar{d}_{R i} d_{L j}^{c} \text {. }
\end{aligned}
$$

From (2) we can get the Feynman rules required to determine the cross-section for the process in Fig. 1. In a process like this, most of the particles in the final state are expected to be lost in the beam pipe, the two exceptions being the quarks resulting from diquark decay. Consequently, the relevant signal is a $p_{T}$ balanced coplanar pair of jets.

The cross-section for the production of a pair of jets through the process in Fig. 1 is given to good accuracy by

$$
\sigma(\hat{s})_{e^{+} e^{-} \rightarrow 2 j e t s}=\int_{x_{\min }}^{1} d x \int_{y_{\min }}^{1} d y f_{p e}(x) f_{p^{\prime} e}(y) \sigma(\hat{s})_{q q^{\prime} \rightarrow q^{\prime \prime} q^{\prime \prime \prime}} \delta(x y s-\hat{s})
$$

In this expression, $f_{p e}(x)$ corresponds to the probability density of finding a parton $p$ carrying a fraction $x$ of the energy of the electrons (or positrons) in the initial beams. 
$\sigma(\hat{s})_{q q^{\prime} \rightarrow q^{\prime \prime} q^{\prime \prime \prime}}$ corresponds to the cross-section for the subprocess in which two on-shell quarks $q$ and $q^{\prime}$, with total energy $\sqrt{\hat{s}}=\sqrt{x y s}$, interact resulting into a diquark that utterly decays to the quarks $q^{\prime \prime}$ and $q^{\prime \prime \prime}$. The lower limits of integration $x_{\min }$ and $y_{\min }$, guarantees the energy required for the production of quarks. Because we are going to consider the five light quarks as massless those limits are actually equal to zero.

The probability $f_{p e}(x)$ results from the convolution of the spectrum of photons emitted by the initial electrons, $f_{\gamma e}(x)$, with $f_{p \gamma}(x)$ the photonic parton distribution function (PDF)

$$
f_{p e}(x)=\int_{0}^{1} d z \int_{0}^{1} d y f_{\gamma e}(z) f_{p \gamma}(y) \delta(z y-x)=\int_{x}^{1} \frac{d z}{z} f_{\gamma e}(z) f_{p \gamma}\left(\frac{x}{z}\right) .
$$

The photon spectrum $f_{\gamma e}$ results from the combination of two effects, namely, bremsstrahlung and beamstrahlung. The photon spectrum resulting from bremsstrahlung for an electron beam with energy $\sqrt{s} / 2$ is [14]

$$
f_{\gamma e}^{b r e m s}(x)=\frac{\alpha_{\mathrm{em}}}{2 \pi}\left[\frac{1+(1-x)^{2}}{x} \ln \frac{s(1-x)^{2}}{m_{e}^{2} x^{2}}+2 m_{e}^{2} x\left(\frac{1}{s(1-x)}-\frac{1-x}{m_{e}^{2} x^{2}}\right)\right] .
$$

The photon spectrum that results from the electromagnetic interaction between the electron and positron beams in the intersection region, is described by a more complicated expression [15]

$$
\begin{aligned}
f_{\gamma e}^{\text {beam }}(x)= & \frac{1}{\Gamma\left(\frac{1}{3}\right)}\left(\frac{2}{3 \Upsilon}\right)^{\frac{1}{3}} x^{-\frac{2}{3}}(1-x)^{-\frac{1}{3}} \exp \left[-\frac{2 x}{3 \Upsilon(1-x)}\right] \\
& \times\left\{\frac{1-\sqrt{\frac{\Upsilon}{24}}}{g(x)}\left[1-\frac{1}{g(x) N_{\gamma}}\left(1-e^{-g(x) N_{\gamma}}\right)\right]\right. \\
& \left.+\sqrt{\frac{\Upsilon}{24}}\left[1-\frac{1}{N_{\gamma}}\left(1-e^{-N_{\gamma}}\right)\right]\right\},
\end{aligned}
$$

The beamstrahlung parameter $\Upsilon$ that characterizes each linear collider is given by,

$$
\Upsilon=\frac{5 r_{e}^{2} E_{e} N}{6 \alpha \sigma_{z}\left(\sigma_{x}+\sigma_{y}\right) m_{e}}
$$

with $E_{e}=\sqrt{s} / 2, r_{e}=\alpha_{\mathrm{em}} / m_{e}=2.818 \cdot 10^{-15}$ the classical electron radius, $\sigma_{x}, \sigma_{y}$ and $\sigma_{z}$ the average size of the particle bunches, and $N$ the number of particles per bunch. Other definitions in (6) are

$$
g(x)=1-\frac{1}{2}\left[(1+x) \sqrt{1+\Upsilon^{\frac{2}{3}}}+1-x\right](1-x)^{\frac{2}{3}},
$$

and the average number of photons per electron irradiated during the electron and positron bunches interaction

$$
N_{\gamma}=\frac{5 \alpha^{2} \sigma_{z} m_{e}}{2 r_{e} E_{e}} \frac{\Upsilon}{\sqrt{1+\Upsilon^{\frac{2}{3}}}}
$$


Because the photon production by bremsstrahlung and beamstrahlung are distinct physical processes the final spectrum is given simply by $f_{\gamma e}(x)=f_{\gamma e}^{\text {brems }}(x)+f_{\gamma e}^{\text {beam }}(x)$. In Fig. 2 we present $f_{\gamma e}(x)$ for TESLA operating at $\sqrt{s}=800 \mathrm{GeV}$. For both TESLA and JLC/NLC at $\sqrt{s}=500 \mathrm{GeV}$ we obtain a similar spectrum, however for JLC/NLC at 1 $\mathrm{TeV}$, the beamstrahlung is significant up to $x \sim 0.7$ instead.

The photonic PDFs were determined by several groups each using distinct physical models and different sets of experimental data [16]. We adopted for our analysis the GRV photonic PDFs [17], calculated at leading order. This choice is based on two facts: (i) according to [16] the GRV parameterization gives one of the best descriptions of the structure function $F_{2}^{\gamma}$ and (ii) this parameterization is valid up to a characteristic scale $Q^{2}=10^{6} \mathrm{GeV}^{2}$. Considering the characteristic scale for the scattering processes equal to $\hat{s} / 4$ implies that it is valid up to $\sqrt{s} \sim 2 \mathrm{TeV}$. Recently, another parameterization [18] was developed, based on an improved model and more data, which fits the data on $F_{2}^{\gamma}$ better than GRV parameterization. However, both parameterizations fits the data almost equally well and the parameterization in [18] presents the limitation of being valid only up to $\sqrt{s} \sim 900 \mathrm{GeV}$, while we are interested in energies up to $1 \mathrm{TeV}$. It is also worth mentioning that for the relatively high values of $Q^{2}$ (the characteristic scale at which the PDFs are evaluated) and $x$ (the energy fraction carried by the parton) that will be important in our search for heavy diquarks, the various parameterizations predict quite similar parton distributions [18]. In Fig. 3 we show $f_{p e}(x)$ for quarks and gluons as expected for TESLA operating at $\sqrt{s}=800 \mathrm{GeV}$, for $Q^{2}=(400 \mathrm{GeV})^{2}$. Similar results hold for TESLA and JLC/NLC.

The last ingredient present in (3) is the cross-section $\sigma(\hat{s})_{q q^{\prime} \rightarrow q^{\prime \prime} q^{\prime \prime \prime}}$. In order to obtain the correct contribution from the subprocess $q q^{\prime} \rightarrow q^{\prime \prime} q^{\prime \prime \prime}$ we have to consider the interference of the $s$-channel production of the diquark with the diagrams involving the gluon exchange in both $t$ and $u$ channels. Denoting these contributions as $a, b$ and $c$, respectively, the differential cross-section for the signal generated by a scalar diquark of mass $M_{S}$ coupling to quarks with strength $\lambda$ can be written as

$$
\begin{aligned}
\frac{d \sigma}{d t} & =\frac{d \sigma(a)}{d t}+\frac{d \sigma(a \times b)}{d t}+\frac{d \sigma(a \times c)}{d t} \\
& =\frac{f_{s} \lambda^{4}}{16 \pi s\left[\left(s-M_{S}^{2}\right)^{2}+M_{S}^{2} \Gamma^{2}\right]}+\frac{f_{s}^{\prime} \lambda^{2} \alpha_{s}\left(s-M_{S}^{2}\right)}{t\left[\left(s-M_{S}^{2}\right)^{2}+M_{S}^{2} \Gamma^{2}\right]}+\frac{f_{s}^{\prime \prime} \lambda^{2} \alpha_{s}\left(s-M_{S}^{2}\right)}{u\left[\left(s-M_{S}^{2}\right)^{2}+M_{S}^{2} \Gamma^{2}\right]}
\end{aligned}
$$

The decay width is

$$
\Gamma=\frac{F_{s} \lambda^{2} M_{S}}{16 \pi}
$$

The factors $f_{s}, f_{s}^{\prime}, f_{s}^{\prime \prime}$ and $F_{s}$ depend upon which of the interactions presented in $(2)$ is considered and incorporate statistical factors associated with the presence of identical fermions in the vertices [19]. The sum and average over colors are incorporated in those factors as well. $F_{s}$ contains the sums over all possible final states for diquark decay. The cross-section (10) was derived assuming massless quarks, a fairly good assumption at the energies we are going to consider. 
At this point we have the freedom to choose which of the interactions from $\mathcal{L}_{s}$ are worth to be analyzed. The idea is to choose a representative interaction whose results can be lately interpreted approximately in terms of the other interactions. For that aim it is helpful to observe in Fig. 3 that $f_{p e}$ for $u$ and $c$ quarks are at least three times bigger than for the other quarks. Because the cross-section (3) involves the product of two $f_{p e}$, we are led to conclude that processes involving $u$ and $c$ quarks are about ten times more likely to occur. For that reason we choose the term $\lambda_{9}^{\prime} S^{\prime \prime i j} \bar{u}_{R i} u_{L j}^{c}$ from $\mathcal{L}_{S}$, which involves $u$ and $c$ quarks. We also consider $\lambda_{9}^{\prime}$, that must be symmetric, diagonal in the flavor space. Consequently the initial and final states in the subprocess in which the diquark is produced involve two $u$ or two $c$ quarks. For comparison, if $\lambda_{9}^{\prime}$ where flavor democratic, we could have 12 instead of 4 different subprocesses. The factors $f_{s}, f_{s}^{\prime}, f_{s}^{\prime \prime}$ and $F_{s}$ are 8 , $8 / 9,8 / 9$ and 4 , respectively, for the interaction we have chosen, for both $u$ and $c$ quarks in the vertices.

\section{BACKGROUND}

The two-jet background receives contributions from the annihilation process $e^{+} e^{-} \rightarrow$ $\gamma, Z \rightarrow q \bar{q}$ and the hard two photon process $e^{+} e^{-} \rightarrow e^{+} e^{-} q \bar{q}$ [20]. The two photon processes can be of type direct, once resolved and twice resolved. The last two processes, despite being higher order in $\alpha_{s}$, contribute significantly at lower energies.

The cross-section for the background generated by the annihilation process is given by

$$
\sigma(\hat{s})_{a n n i h}=\sum_{q} \int_{0}^{1} d x \int_{0}^{1} d y f_{e e}(x) f_{e e}(y) \sigma(\hat{s})_{e^{+} e^{-} \rightarrow q \bar{q}} \delta(x y s-\hat{s}),
$$

where $f_{e e}(x)$ is the electron (and positron) spectrum, the probability density that we get an electron with a fraction $x$ of the initial electron energy in the beams, and the sum runs over all possible quarks in the final state. $\sigma(\hat{s})_{e^{+} e^{-} \rightarrow q \bar{q}}$ corresponds to the cross-section for the process $e^{+} e^{-} \rightarrow \gamma, Z \rightarrow q \bar{q}$. $f_{e e}$ results from the convolution of the beamstrahlung and the initial state radiation (ISR) spectra

$$
f_{e e}(x)=\int_{x}^{1} \frac{d z}{z} f_{e e}^{I S R}(z) f_{e e}^{b e a m}\left(\frac{x}{z}\right)
$$

We use the following analytical expression for $f_{e e}^{I S R}(x)[21]$,

$$
f_{e e}^{I S R}(x)=\frac{\beta}{2}(1-x)^{\frac{\beta}{2}-1}\left(1+\frac{3}{8} \beta\right)-\frac{1}{4} \beta(1+x),
$$

where $\beta$ is defined as,

$$
\beta=\frac{2 \alpha_{\mathrm{em}}}{\pi}\left[\ln \left(\frac{s}{m_{e}^{2}}\right)-1\right] .
$$

The spectrum $f_{\text {ee }}^{\text {beam }}$ was evaluated numerically using CIRCE [22] a FORTRAN routine containing parameterizations for electron, positron and photon spectra resulting from beamstrahlung at the future $e^{+} e^{-}$colliders. 
The cross-section for the background generated by two hard photon processes is calculated from

$$
\sigma(\hat{s})_{2 \gamma}=\sum_{i j} \sum_{p} \int_{0}^{1} d x \int_{0}^{1} d y f_{i e}(x) f_{j e}(y) \sigma(\hat{s})_{i j \rightarrow 2 j e t s} \delta(x y s-\hat{s}),
$$

where the indexes $i, j=\gamma$ or $p$. The sum over $i$ and $j$ corresponds to the sum over direct, once and twice resolved processes and the sum over $p$ remind us that we have to sum over subprocesses involving different initial and final partons. In total we have to consider one direct process, and 2 once resolved, and 8 twice resolved subprocesses. The cross-sections for all such processes can be found in [23]. The five lighter quarks are assumed massless throughout our analysis and the top quark, of course, is not considered as a source of background.

\section{RESULTS}

In the previous sections we presented all the theoretical and computational tools required for our analysis. We used them to determine the expected significance of the signal over background for collider parameters as given in [9], for TESLA operating at $500 \mathrm{GeV}$ and $800 \mathrm{GeV}$, and as given in [24] for NLC operating at $500 \mathrm{GeV}$ and $1 \mathrm{TeV}$. Because the designs for JLC and NLC tends to converge, specially with respect to the parameters relevant for the beamstrahlung effect, we can in fact assume that our results are valid for JLC/NLC design. We show in Table I the relevant parameters for the calculation of beamstrahlung.

The significance of signal over background is defined as,

$$
\sigma_{S}=\frac{S}{\sqrt{B}}
$$

where $S$ is the number of expected two-jet events attributed to the resonant production of diquarks, and $B$ is the number of two-jet events resulting from the standard model alone. The signal generated by a diquark of mass $M_{S}$ and decay rate $\Gamma$ is calculated integrating the cross-section in the two-jet invariant mass $(M)$ interval $M_{S}-\Gamma<M<M_{S}+\Gamma$, which embraces approximately $95 \%$ of the events around the resonance. For a realistic analysis of the contribution of the background we considered the energy resolution of the hadronic calorimeter. For TESLA detector it reads,

$$
\frac{\delta E}{E} \leq \frac{0.5}{\sqrt{E}}+0.04
$$

The same energy resolution was assumed for JLC/NLC. The corresponding two-jet invariant mass resolution is given approximately (we assume it to be exact in our calculations) by $\delta M=0.5 \sqrt{M}+0.02828 M$. The background B is calculated by integrating the crosssection in the range $M_{S}-\Delta M<M<M_{S}+\Delta M$, with $\Delta M=\max (\Gamma, \delta M)$.

The significance $(17)$, scales with the collider luminosity as $\sqrt{\mathcal{L}}$. Consequently, high luminosities are desirable for a good signal over background discrimination. Presently, TESLA is expected to deliver the highest luminosities, $500 \mathrm{fb}^{-1} /$ year, while the luminosity 
for JLC/NLC is planned to be in the range 100-200 $\mathrm{fb}^{-1} /$ year. With those luminosities in mind we determined the significance for the following total integrated luminosities: $\mathcal{L}=100,300$ and $500 \mathrm{fb}^{-1}$ for both collider designs and additionally $\mathcal{L}=2000 \mathrm{fb}^{-1}$ for TESLA.

In determining $\sigma_{S}$ we considered some cuts on the pseudo-rapidity $\eta$ and the transverse momentum $p_{T}$ of the jets. We worked $\sigma_{S}$ out for $|\eta|<1$ and 2 , and $\left|p_{T}\right|>15 \mathrm{GeV}$, for both jets. This cut on $p_{T}$ is about the minimum required to guarantee that the assumption of massless quarks is valid for all quarks, including the bottom quark, because it implies an adequate minimum energy for the jets. It also guarantees the validity of the PDF parameterization and eliminates considerable amount of background. As we discuss in more detail later, higher $p_{T}$ cuts did not improve $\sigma_{S}$ significantly, while reducing the absolute number of signal events to undesirably low values. Our analysis is also limited to the search for diquarks with masses above $100 \mathrm{GeV}$. Lighter Diquarks could in principle be pair produced by LEP, which reached more than $200 \mathrm{GeV}$ as its center of mass energy. Here we are interested in what LEP could not reach.

In Fig. 5 we present $S / \sqrt{B}$ as a function of the diquark mass, $M_{S}$, for TESLA operating at $500 \mathrm{GeV}$. The solid and dotted lines are for $|\eta|<1$ and 2, respectively. The eight upper curves, both solid and dotted, correspond to a coupling with strength given by $\alpha=\lambda^{2} / 4 \pi=0.1$, while the eight lower curves are for $\alpha=0.01$. For each $\alpha$ and cut on $\eta$ there are four curves corresponding to the integrated luminosities $\mathcal{L}=$ $100,300,500$ and $2000 \mathrm{fb}^{-1}$. The curves for $\alpha=0.01$ where scaled by a factor 0.1 in order to clearly separate them from the curves for $\alpha=0.1$. The straight dashed lines correspond to $S / \sqrt{B}=3$, the minimum requirement for signal observability. The choice $\alpha=0.1$ corresponds approximately to the maximum acceptable value to keep higher order contributions under control. The coupling $\alpha=0.01$ is approximately the minimum value for which the signal significance is worth to be analyzed. For smaller values of $\alpha$ the signal significance is much smaller than 3 for masses greater than $\sqrt{s} / 2$. Fig. 6 shows the same curves for TESLA operating at $800 \mathrm{GeV}$. The results for JLC/NLC operating at $500 \mathrm{GeV}$ and $1 \mathrm{TeV}$ are presented in Figs. 7 and 8 for $\mathcal{L}=100,300$ and $500 \mathrm{fb}^{-1}$.

\section{FINAL DISCUSSION AND CONCLUSION}

As already mentioned at the Introduction the Tevatron collider has set limits on the masses of a class of scalar diquarks, $290<M_{\phi_{E_{6}}}<420 \mathrm{GeV}$ which are expected to be approximately valid for other scalar diquarks. There also exist indirect bounds imposed on Yukawa coupling constants for quarks and diquarks. Stringent indirect bounds for couplings involving the second and third generations where set from the limits on branching ratio for the inclusive decay $B \rightarrow X_{s} \gamma$ [25]. Looser bounds on couplings involving other generations come from the analysis of precision LEP data [26], neutronantineutron oscillation [27] and rare nucleon and meson decay [28]. The looser bounds generally permit couplings of the order one as required for $\alpha=0.1(\lambda \sim 1.25)$ the higher value we admitted in our analysis.

It is exactly for relatively high values of the Yukawa coupling constant that we may expect the resonant production of diquarks to be useful. This conclusion can be draught from the curves for $\alpha=0.1$ in Figs. 5, 6, 7 and 8. For couplings smaller by just one order 
of magnitude $(\alpha=0.01)$ the resonant production does not produce any interesting result. Is is worth mentioning at this point that for $\alpha=0.1$ the number of signal events is always of order of a few tens or more for $\sigma_{S}>3$, resulting in good statistics. Another conclusion that can be draught is that the best signal over background relation in the parameter region satisfying $\sigma_{S}>3$ and $M_{S}>\sqrt{s} / 2$ is obtained considering the cut $|\eta|<1$. However we can note from the results for the signal significance that the curves for $|\eta|<1$ are not always above the corresponding curves for $|\eta|<2$. This behavior for $\sigma_{s}$ can be attributed to the distinct angular distributions for the background generated through annihilation and for the background generated by two hard photon processes. The two hard photon processes prevail at energies below $\sqrt{s} / 2$ while the annihilation prevails at higher energies. This partially explains the behavior of the curves. An additional aspect of the background has to be taken into account, the fact that once and twice resolved processes initiated by gluons, that alter significantly the angular distribution, are more important at lower

energies. Altogether, we have the elements that imply the relatively complex behavior of the curves for signal significance.

A final comment on the $p_{T}$ cuts. We have tested cuts on $p_{T}$ higher than $15 \mathrm{GeV}$, however they did not improve the signal over background relation significantly. This can be explained by the fact that the dependence on $p_{T}$ for the signal is almost the same as the one for the background generated by hard two photon processes and the background from annihilation is almost constant with respect to $p_{T}$.

We conclude, considering the present analysis and the previous works on the search for diquarks at the next generation of linear colliders, that diquarks with masses above $\sqrt{s} / 2$ are unlikely to be seen at such machines, unless new production channels are devised and prove to be sufficiently sensitive to the presence of those particles. The only exception are diquarks with relatively strong Yukawa coupling constants, that means $\lambda \sim 1$, with the additional requirement of coupling to $u$ and $c$ quarks.

\section{ACKNOWLEDGMENTS}

This work was supported by Fundação de Amparo a Pesquisa do Estado de São Paulo (FAPESP). 


\section{REFERENCES}

[1] J. L. Hewett, T. G. Rizzo, Phys. Rep. 183, 193 (1989).

[2] J. Wudka, Phys. Lett. B 167, 337 (1986).

[3] P. H. Frampton, S. L. Glashow, T. Yoshikawa, Phys. Rev. Lett. 87, 011801-1 (2001).

[4] G. D'Ambrosio, D.-N. Gao, Phy. Lett. B 513, 123 (2001).

[5] E. N. Argyres et al., Phys. Lett. B 237, 577 (1990); A. P. Contogouris, G. Katsilieris, O. Korakianitis, S. D. P. Vlassopulos, Phys. Lett. B 287, 203 (1992); D. Schaile, P. Zerwas, Proceedings of the Workshop on Physics at Future Accelerators, CERN yellow report 87-07, Vol. II, 251 (1992).

[6] T. G. Rizzo, Z. Phys. C 43, 223 (1989).

[7] V. D. Angelopoulos et al., Nucl. Phys. B 292, 59 (1987); E. N. Argyres, C. G. Papadopoulos, S. D. P. Vlassopulos, Phys. Lett. B 202, 425 (1988).

[8] S. Atag, O. Çakir, S. Sultansoy, Phys. Rev. D 59, 015008-1 (1998); E. Arik et al., JHEP 0209, 24 (2002).

[9] TESLA - Technical Design Report, available at the URL http://tesla.desy.de/new_pages/TDR_CD/start.html.

[10] General and technical information about the JLC/NLC project is available at the URLs http://www-project.slac.stanford.edu/nlc/home.html and http://lcdev.kek.jp/.

[11] T. Dorigo, FERMILAB-CONF-97-281-E, CDF-PUB-EXOTIC-PUBLIC-4238, 1997 (unpublished).

[12] E. M. Gregores, A. Gusso, S. F. Novaes, Phys. Rev. D 64, 015004 (2001).

[13] J. F. Nieves, Nucl. Phys. B 189, 182 (1981).

[14] D. de Florian, S. Frixione, Phys. Lett. B 457, 236 (1999).

[15] P. Chen, Phys. Rev. D 46, 1186 (1992).

[16] For a recent review on photonic parton distribution functions see M. Krawczyk, A. Zembrzuski, M. Staszel, Phys. Rept. 345, 265 (2001).

[17] M. Glück, E. Reya, A. Vogt, Phys. Rev. D 46, 1973 (1992).

[18] F. Cornet, P. Jankowsk, M. Krawczyk, A. Lorca, Phys. Rev. D 68, 014010 (2003).

[19] S. R. Moore, K. Whisnant, Bing-Lin Young, Phys. Rev. D 31, 105 (1985).

[20] M. Drees, R. M. Godbole, Z. Phys. C 59, 591 (1993).

[21] E. A. Kuraev, V. S. Fadin, Sov. J. Nucl. Phys. 41, 466 (1985).

[22] T. Ohl, Comput. Phys. Commun. 101, 269 (1997).

[23] E. Leader, E. Predazzi, Introduction to Gauge Theories and Modern Particle Physics, Vol. 2, 1st edn. (Cambridge University Press, 1996).

[24] N. Phinney, SLAC-PUB-9267, 2002 (unpublished).

[25] D. Chakraverty, D. Choudury, Phys. Rev. D 63, 075009 (2001).

[26] G. Bhattacharyya, D. Choudhury, K. Sridhar, Phys. Lett. B 355, 193 (1995).

[27] J. L. Goity, M. Sher, Phys. Lett. B 346, 69 (1995).

[28] C. E. Carlson, P. Roy, M. Sher, Phys. Lett. B 357, 99 (1995). 


\section{FIGURES}

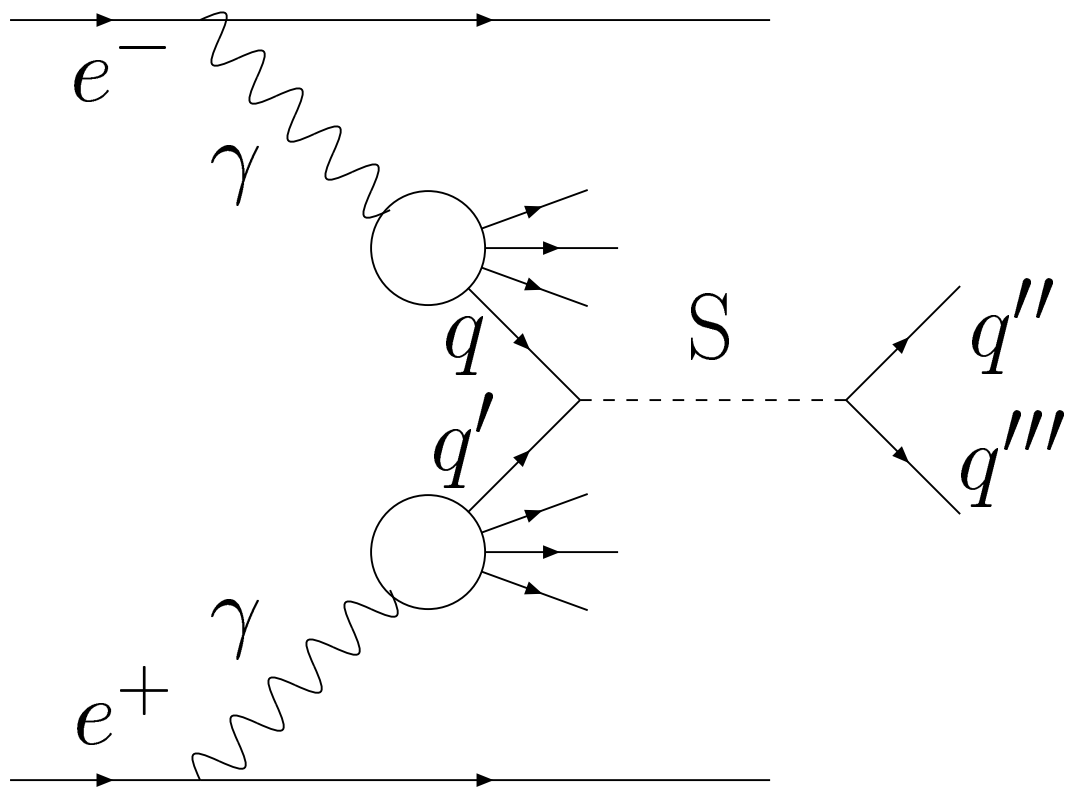

FIG. 1. Schematic representation of the process leading to the resonant production of diquarks in $e^{+} e^{-}$colliders.

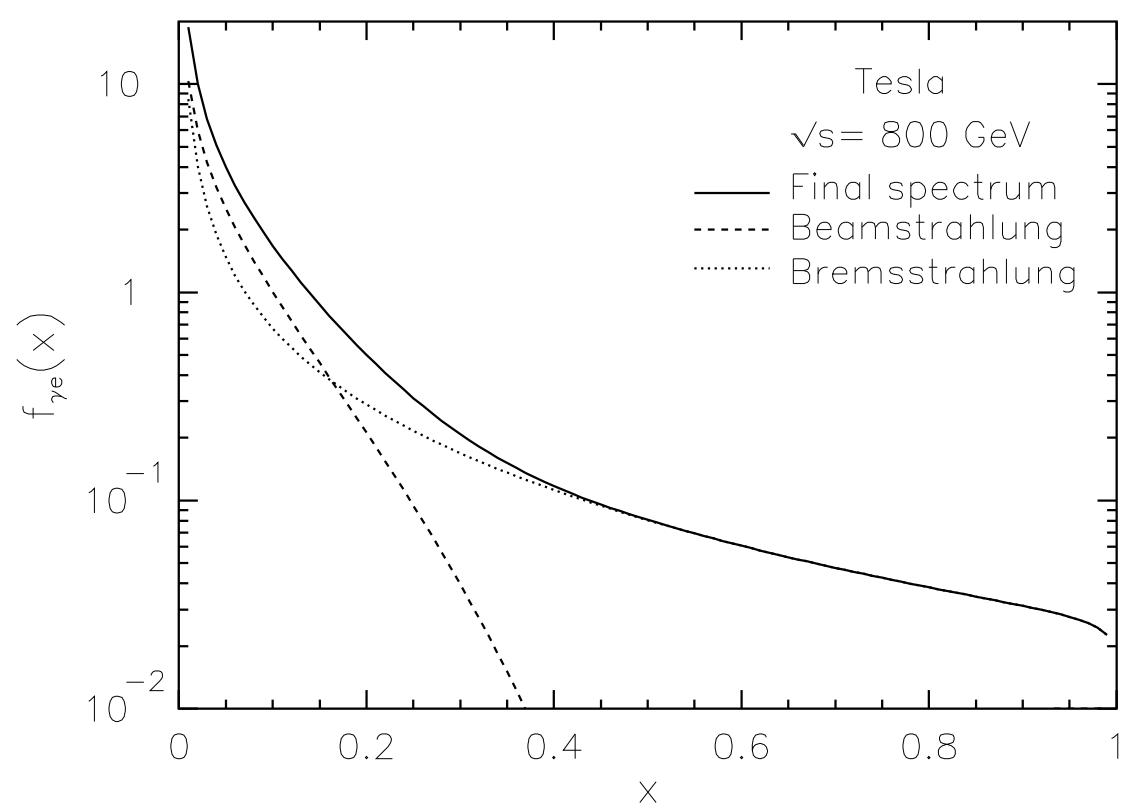

FIG. 2. The probability density $f_{\gamma e}$ for TESLA operating at $\sqrt{s}=800 \mathrm{GeV}$. 


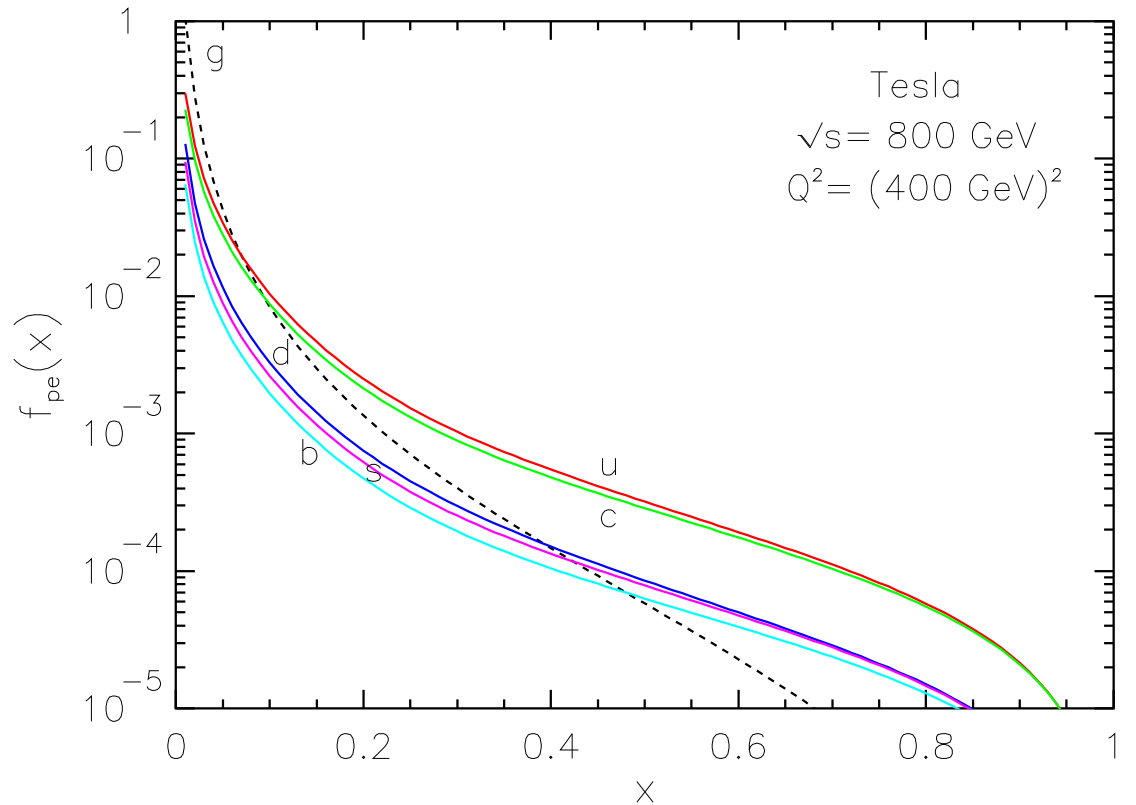

FIG. 3. The probability density $f_{p e}$ for quarks and gluons for TESLA operating at $\sqrt{s}=800$ $\mathrm{GeV}$ and an energy scale $Q^{2}=(400 \mathrm{GeV})^{2}$.

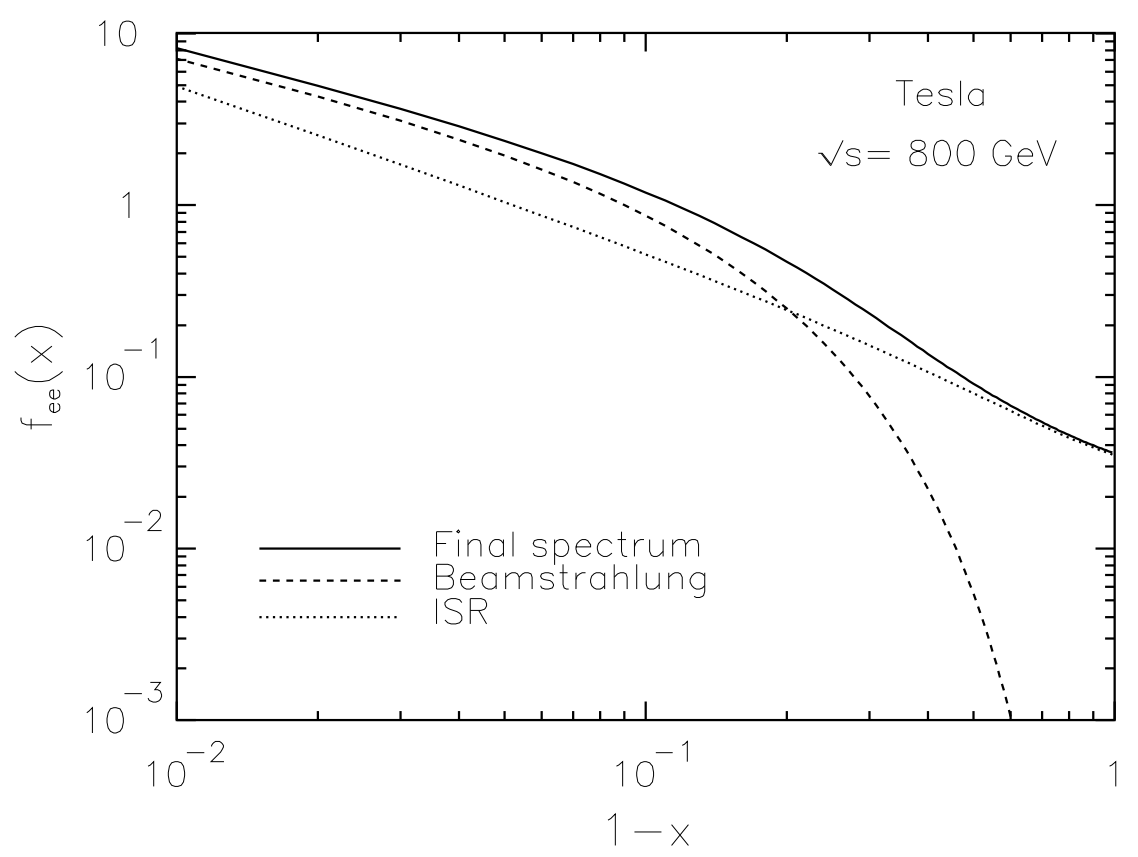

FIG. 4. The probability density $f_{e e}$ for TESLA operating at $\sqrt{s}=800 \mathrm{GeV}$. 


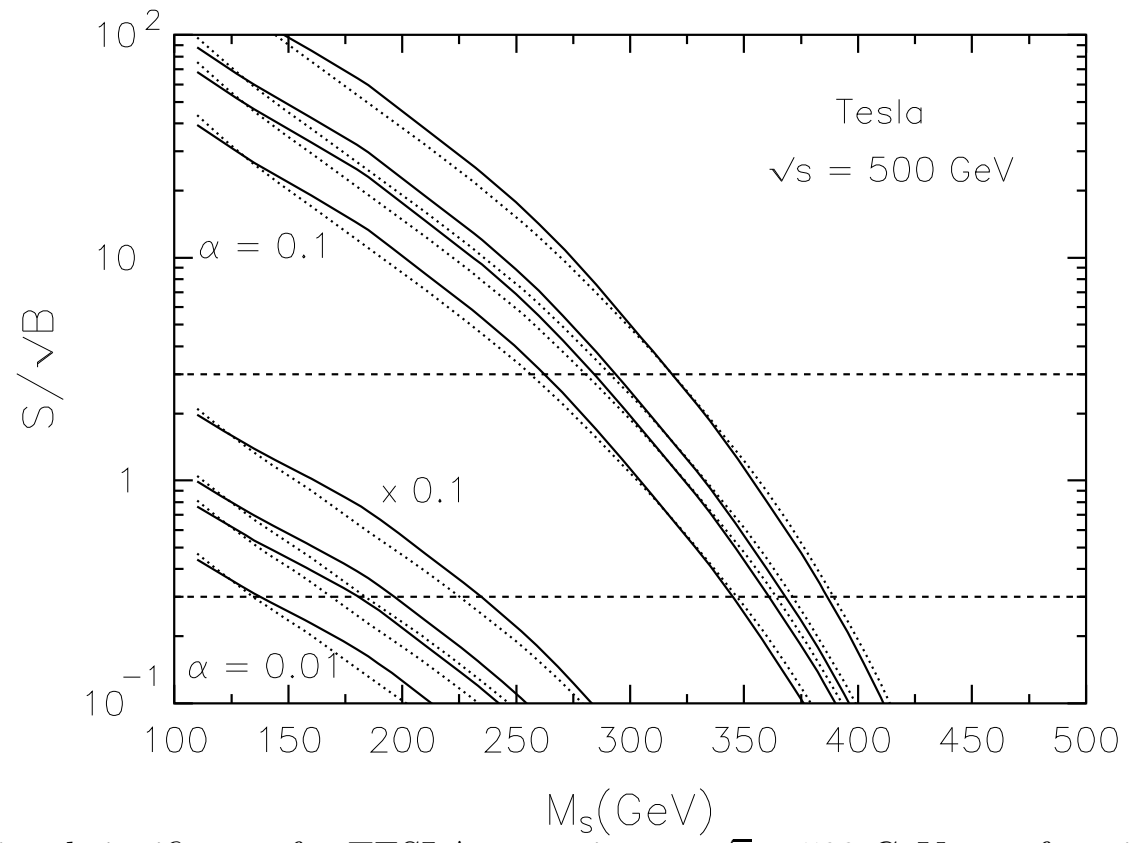

FIG. 5. Signal significance for TESLA operating at $\sqrt{s}=500 \mathrm{GeV}$ as a function of diquark mass, $M_{S}$. Solid and dotted lines correspond to $|\eta|<1$ and 2, respectively. For all curves $\left|p_{T}\right|>15 \mathrm{GeV}$.

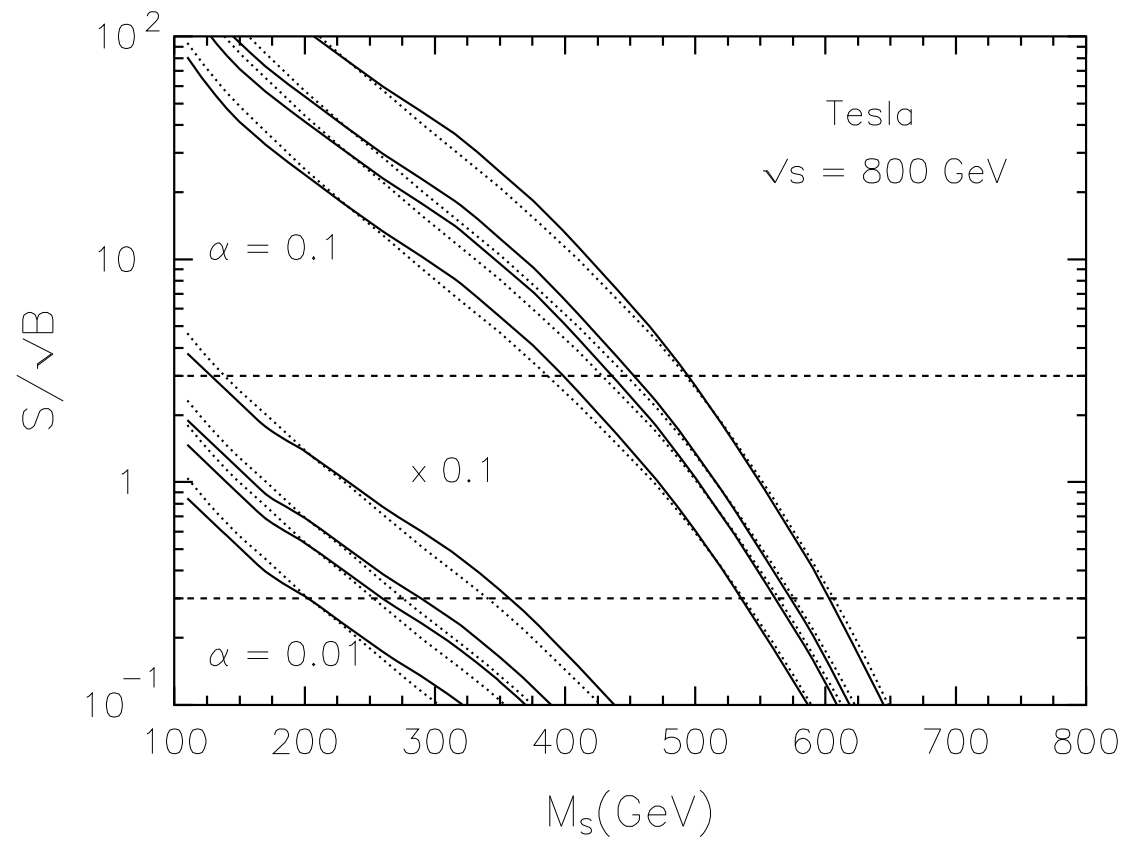

FIG. 6. The same as Fig. 5 for TESLA operating at $\sqrt{s}=800 \mathrm{GeV}$. 


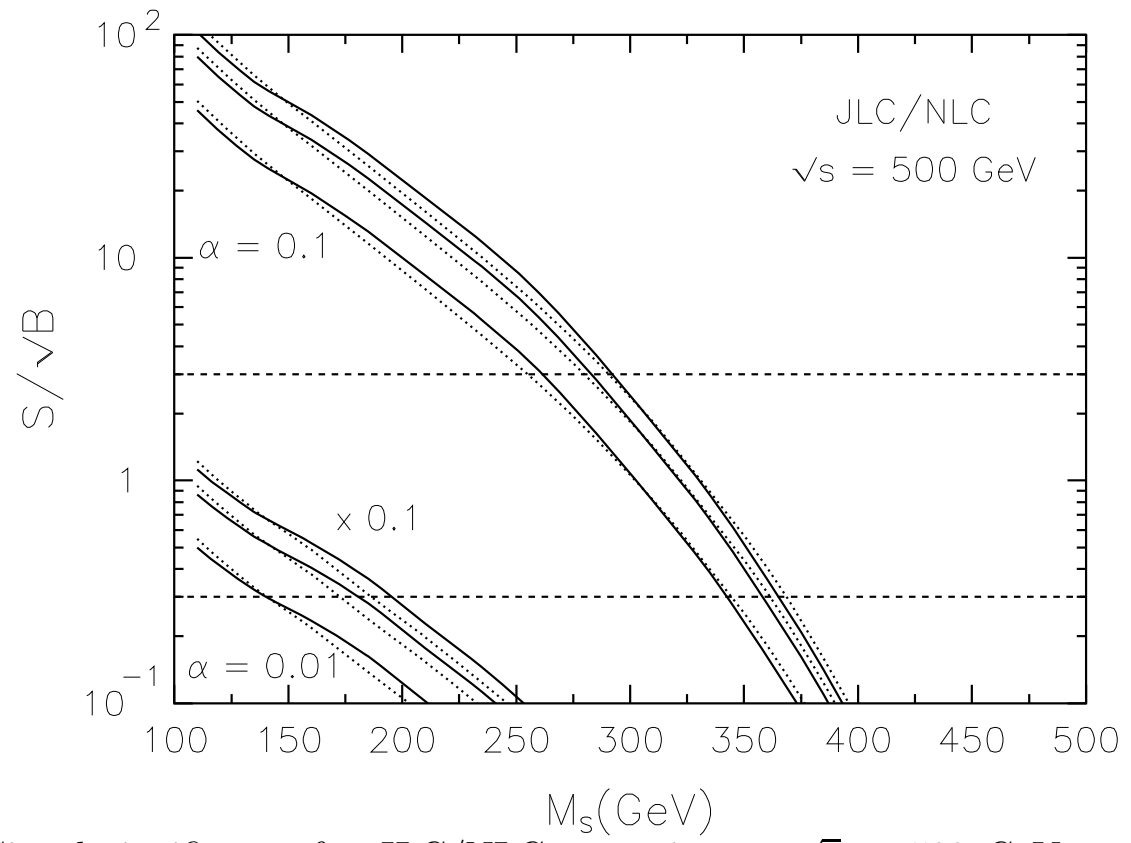

FIG. 7. Signal significance for JLC/NLC operating at $\sqrt{s}=500 \mathrm{GeV}$ as a function of diquark mass, $M_{S}$. Solid and dotted lines correspond to $|\eta|<1$ and 2, respectively. For all curves $\left|p_{T}\right|>15 \mathrm{GeV}$.

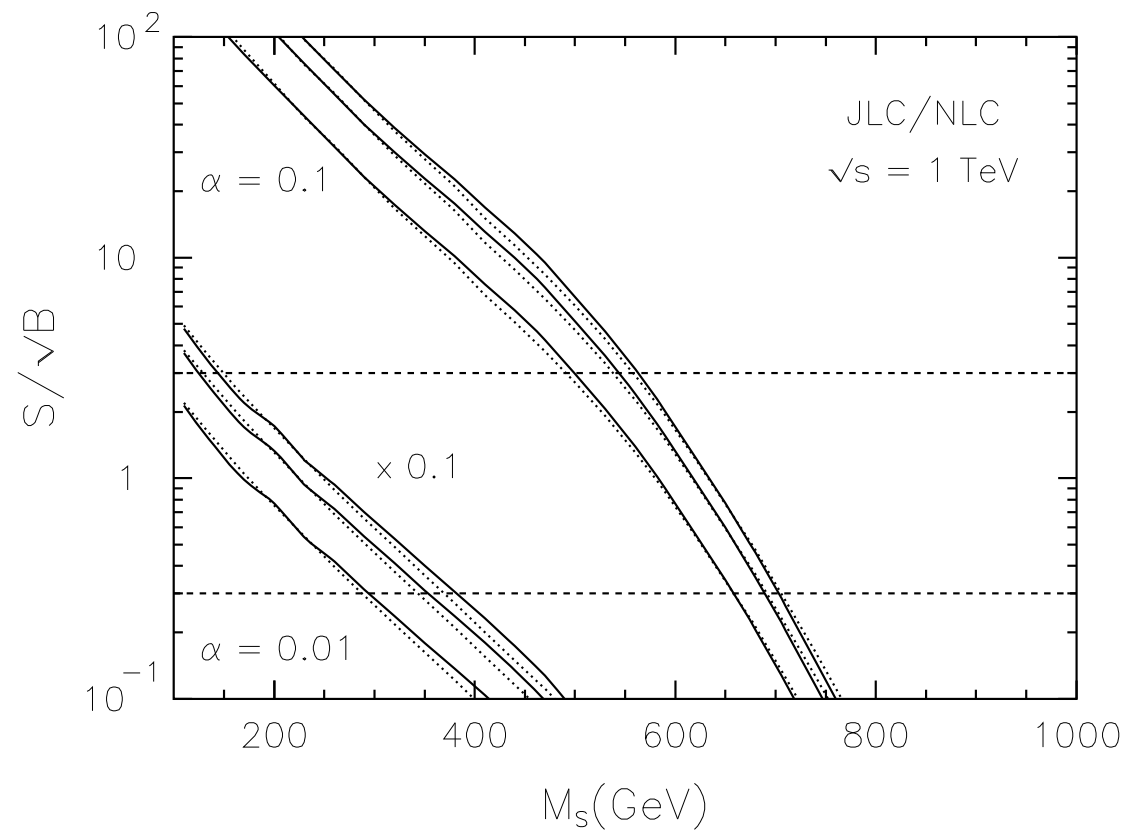

FIG. 8. The same as Fig. 7 for JLC/NLC operating at $\sqrt{s}=1 \mathrm{TeV}$. 


\section{TABLES}

TABLE I. Collider parameters relevant for the calculation of beamstrahlung. $N$ is the number of particles per bunch, $\sigma_{x, y, z}$ are the mean bunch dimensions ( $z$ is the beam axis), $\Upsilon$ is the beamstrahlung parameter and $N_{\gamma}$ the average number of photons per electron.

\begin{tabular}{|c|c|c|c|c|c|}
\hline & Collider & TESLA & TESLA & JLC/NLC & $\mathrm{JLC} / \mathrm{NLC}$ \\
\hline Parameter & & $500 \mathrm{GeV}$ & $800 \mathrm{GeV}$ & $500 \mathrm{GeV}$ & $1 \mathrm{TeV}$ \\
\hline$N\left[10^{10}\right]$ & & 2.0 & 1.4 & 0.75 & 0.75 \\
\hline$\sigma_{x}[\mathrm{~nm}]$ & & 553 & 391 & 245 & 190 \\
\hline$\sigma_{y}[\mathrm{~nm}]$ & & 5 & 2.8 & 2.7 & 2.1 \\
\hline$\sigma_{z}[\mu \mathrm{m}]$ & & 300 & 300 & 110 & 110 \\
\hline$\Upsilon$ & & 0.053 & 0.084 & 0.122 & 0.315 \\
\hline$N_{\gamma}$ & & 1.44 & 1.39 & 1.16 & 1.38 \\
\hline
\end{tabular}

